\title{
The Prognostic Role and Nomogram Establishment of a Novel Prognostic Score Combining with Fibrinogen and Albumin Levels in Patients with WHO Grade II/III Gliomas
}

Tianshu Jia'

Rui Zhang'

Fanfei Kong ${ }^{2}$

Qianjiao Zhang ${ }^{3}$

Zhuo Xi (D)'

'Department of Neurosurgery, Shengjing Hospital of China Medical University, Shenyang, People's Republic of China: ${ }^{2}$ Department of Obstetrics and Gynecology, Shengjing Hospital of China Medical University, Shenyang, People's Republic of China; ${ }^{3}$ Pain Department, The People's Hospital of Liaoning Province, Shenyang, People's Republic of China
Correspondence: Zhuo Xi

Department of Neurosurgery, Shengjing Hospital of China Medical University, No. 36 Sanhao Street, Shenyang, People's

Republic of China

Tel +86 18940258425

Email neurosurgeon-xz@hotmail.com
Purpose: World Health Organization (WHO) Grades II and III gliomas [also known as low grade gliomas (LGGs)] displayed different malignant behaviors and survival outcomes compared to Grade IV gliomas. This study aimed to identify the prognostic predictive value of a novel cumulative prognostic score [combined with fibrinogen and albumin levels (FA score)], establish and validate a point-based nomogram in LGG patients.

Patients and Methods: A total of 91 patients who underwent total glioma resection at Shengjing Hospital of China Medical University between 2011 and 2013 were enrolled to establish a prognostic nomogram. All patients were histologically diagnosed as grades II/III, and never received radiotherapy or chemotherapy before surgery. Data collection included patient characteristics, clinicopathological factors, and preoperative hematology results. The performance of the nomogram was subsequently validated by the concordance index (c-index), calibration curve, and receiver operating characteristic (ROC) curve.

Results: The FA score was negatively associated with the overall survival (OS) of LGG patients $(\mathrm{p}<0.001)$. The results of multivariate analysis showed that FA score $[\mathrm{p}=0.006$, $\mathrm{HR}=1.92,95 \%$ confidence interval $(\mathrm{CI}): 1.21-3.05]$, age $(\mathrm{p}=0.002, \mathrm{HR}=3.014,95 \%$ CI:1.52-5.97), and white blood count ( $\mathrm{p}<0.001, \mathrm{HR}=4.24,95 \% \mathrm{CI}: 2.08-8.67)$ were independent prognostic factors for overall survival (OS). The study established a nomogram to predict OS with a c-index of 0.783 (95\% CI, 0.72-0.84).

Conclusion: FA score might be a potential prognostic biomarker for LGG patients, and a reliable point-based nomogram will help clinicians to decide on the best treatment plans.

Keywords: prognostics, fibrinogen, albumin, low-grade gliomas, nomogram

\section{Introduction}

Glioma is the most commonly occurring type of malignant primary brain tumor. ${ }^{1}$ World Health Organization (WHO) Grades II and III gliomas were recently classified as low-grade gliomas (LGGs). ${ }^{2,3}$ LGGs typically appear non-enhancing on magnetic resonance imaging scans, while glioblastomas (GBMs) usually show enhanced signals. ${ }^{4}$ It is difficult to distinguish LGGs from GBMs only by histopathology, because they can both show microvascular proliferations, the presence of necrosis, etc. ${ }^{5,6}$ LGGs, as opposed to GBMs, display significantly different prognostic outcomes. ${ }^{7}$ Histopathology alone is often insufficient to make an accurate prognosis, and so it is necessary to identify prognostic risk factors for LGG patients. 
The histopathological characteristics, age at diagnosis, and performance status are traditional prognostic indicators for gliomas. ${ }^{8,9}$ The increasing age and poor performance status (Karnofsky performance score $\leq 80$ ) are definitive negative prognostic factors. ${ }^{8}$ Notably, emerging evidence has indicated that the systemic inflammation level and nutritional status of patients indicated their prognostic values in various solid tumors, including gliomas. ${ }^{10-13}$ The cancerassociated inflammation and accumulation of inflammatory cells around cancer cells have been associated with cancer occurrence and progression. ${ }^{14}$ Matsuda and co-workers first proposed a novel cumulative prognostic score (FA score) in esophageal cancer, which was combined with fibrinogen and albumin levels. They reported that patients with a high preoperative FA score showed shorter disease-free survival and overall survival. ${ }^{11}$ Subsequently, $\mathrm{He}$ and co-workers also evaluated the negative prognostic role of high FA scores with overall survival in high grade gliomas (HGGs). ${ }^{12}$ However, some issues remained unexplained. For example, only HGG patients were analyzed in previous studies, excluding LGG patients. More importantly, only the FA score was included, and there was no other blood-derived indicator containing a systemic inflammation index. ${ }^{12}$ The aim of the present study was therefore to identify the prognostic predictive values of FA scores, which represented the other blood-derived and clinicopathological characteristics in patients with LGGs. To the best of our knowledge, a predictive nomogram for prognoses of LGG patients based on these biomarkers and clinicopathological characteristics has not been previously reported.

\section{Methods and Materials Study Population}

Patients with histologically diagnosed Grade II/III gliomas who underwent curative gross total resection at the Shengjing Hospital of China Medical University between 2011 and 2013 were retrospectively reviewed. The inclusion criteria of patients were as follows: 1) age $\geq 18$ years, 2) Grade II-III gliomas confirmed by histopathology, 3) the surgical procedure involved gross total resection, and 4) the availability of clinicopathological and biochemical data. None of these patients received radiotherapy or chemotherapy before surgery. The study was approved by the Ethics Committee at Shengjing Hospital of China Medical University (2017PS211K). All patients in the study signed informed consent forms. This study was conducted in accordance with the Declaration of Helsinki.

\section{Data Collection}

The original clinical data were collected from hospital medical records, including patient age, gender, ECOG PS (Eastern Cooperative Oncology Group performance status), tumor location, maximum tumor diameter and WHO grade. Preoperative blood test indicators included the white blood cell count, hemoglobin, platelet count, neutrophil-to-lymphocyte ratio, platelet-to- lymphocyte ratio, total protein, albumin, alanine aminotransferase (ALT), aspartate aminotransferase, total bilirubin, and direct bilirubin levels. The primary end point of this study was overall survival (OS). OS was counted from the surgery time to the time of death or the last follow-up visit. Patients' deaths were directly related to gliomas.

\section{FA Score and AFR}

The fibrinogen cut-off value was defined as $2.5 \mathrm{~g} / \mathrm{l}$ and the cut-off value of albumin was $38.3 \mathrm{~g} / 1$, which were determined by the X-tile program. Patients with elevated fibrinogen and decreased albumin levels were scored 2 points, and only one of the abnormal patients was scored 1 point. None of these abnormalities were scored $0 .{ }^{11}$ The patients were divided into three groups based on the best cut-off values of the albumin/fibrinogen ratio (AFR).

\section{Nomogram Development and Validation}

For developing a nomogram, the clinicopathological variables that achieved a significance level of $p<0.2$ using univariate analyses were entered into multivariate analyses for screening as independent risk factors using the Cox regression model. Based on all independent prognostic factors, a nomogram was constructed to allow for a visualized estimate of individual OS probabilities at 1 and 3 years. The nomogram was validated by the bootstrap-corrected Harrell's concordance index (c-index), calibration curves and time-dependent ROC curves.

\section{Statistical Analysis}

Laboratory variables were recorded as continuous variables and dichotomized based on the cut-off values determined by the X-tile program (http://www.tissuear ray.org/rimmlab) ${ }^{15,16}$ A value of $p<0.05$ was considered significant. The Kaplan-Meier survival method was 
used to produce the survival curves. Statistical analyses (SPSS, Chicago, IL, USA) and R software version were performed using SPSS 23.0 software package 3.3.1 (http://www.r-project.org).

Table I Relationships Between Patient Demographics and Clinicopathological Characteristics and FA Score with Characteristics

\begin{tabular}{|c|c|c|c|c|c|c|}
\hline Characteristics & $\mathbf{N}$ & Percent/Mean (SD) & FA Score $0(n=35)$ & FA Score I $(n=42)$ & FA Score $2(n=14)$ & $P$ value \\
\hline Age, years & 91 & $45.42(|4.6|)$ & $39.74 \pm 12.06$ & $46.83 \pm 14.50$ & $55.36 \pm 15.3$ & 0.002 \\
\hline Gender & & & & & & 0.648 \\
\hline Male & 56 & $61.5 \%$ & $20(57.1)$ & $26(61.9)$ & $10(71.4)$ & \\
\hline Female & 35 & $38.5 \%$ & $15(42.9)$ & $16(38.1)$ & $4(28.6)$ & \\
\hline ECOG PS & & & & & & 0.467 \\
\hline $0-1$ & 64 & $70.3 \%$ & $27(77.1)$ & $27(64.3)$ & $10(71.4)$ & \\
\hline $2-3$ & 27 & $29.7 \%$ & $8(22.9)$ & $15(35.7)$ & $4(28.6)$ & \\
\hline Grade & & & & & & 0.082 \\
\hline II & 60 & $65.9 \%$ & $28(80.0)$ & $24(57.1)$ & $8(57.1)$ & \\
\hline III & 31 & $34.1 \%$ & $7(20.0)$ & $18(42.9)$ & $6(42.9)$ & \\
\hline Location & & & & & & 0.574 \\
\hline Left brain & 38 & $41.8 \%$ & $13(37.1)$ & $20(47.6)$ & $5(35.7)$ & \\
\hline Right brain & 53 & $58.2 \%$ & $22(62.9)$ & $22(52.4)$ & $9(64.3)$ & \\
\hline Max tumor & 87 & $4.822(1.90)$ & $4.62 \pm 1.75$ & $4.96 \pm 2.03$ & $4.93 \pm 1.97$ & 0.735 \\
\hline Adjuvant treatment & & & & & & 0.920 \\
\hline None & 35 & & 13 & 16 & 6 & \\
\hline Radiotherpy & 9 & & 4 & 4 & I & \\
\hline TMZ & 16 & & 8 & 7 & I & \\
\hline Radiotherpy +TMZ & 13 & & 3 & 7 & 3 & \\
\hline Unknown & 18 & & 7 & 8 & 3 & \\
\hline WBC, $10^{9} / \mathrm{L}$ & 91 & $7.48(3.28)$ & $7.04 \pm 2.87$ & $7.62 \pm 2.92$ & $8.19 \pm 5.00$ & 0.509 \\
\hline HGB, g/L & 91 & $137.0 \mid(15.57)$ & $137.7 \pm 13.33$ & $136.99 \pm 16.94$ & $|35.37 \pm| 5.57$ & 0.896 \\
\hline PLT, $10^{9} / \mathrm{L}$ & 91 & $212.91(57.32)$ & $205.64 \pm 64.52$ & $221.65 \pm 47.23$ & $204.86 \pm 66.06$ & 0.408 \\
\hline NLR & 91 & $3.4 I(3.37)$ & $2.79 \pm 1.81$ & $3.43 \pm 3.16$ & $4.90 \pm 5.91$ & 0.140 \\
\hline PLR & 91 & $131.79(54.77)$ & $|25.58 \pm 6| .14$ & $134.16 \pm 50.73$ & $|40.19 \pm 5| .74$ & 0.656 \\
\hline $\mathrm{TP}, \mathrm{g} / \mathrm{L}$ & 89 & $67.49(5.58)$ & $68.23 \pm 4.66$ & $68.47 \pm 5.94$ & $62.82 \pm 4.53$ & 0.002 \\
\hline ALB, $g / L$ & 91 & $41.07(4.13)$ & $42.71 \pm 2.69$ & $41.52 \pm 4.03$ & $35.67 \pm 2.95$ & $<0.001$ \\
\hline ALT, U/L & 86 & $22.52(20.84)$ & $27.89 \pm 28.15$ & $20.05 \pm \mid 4.01$ & $14.92 \pm 7.29$ & 0.107 \\
\hline AST, U/L & 89 & $17.27(10.29)$ & $19.57 \pm 14.64$ & $16.72 \pm 6.09$ & $13.07 \pm 3.29$ & 0.122 \\
\hline TBIL, umol/L & 89 & $10.11(4.52)$ & $10.96 \pm 4.80$ & $10.01 \pm 4.38$ & $8.31 \pm 3.87$ & 0.176 \\
\hline DBIL, umol/L & 89 & $3.88(2.01)$ & $4.18 \pm 1.86$ & $3.65 \pm 1.85$ & $3.75 \pm 2.78$ & 0.516 \\
\hline Fibrinogen, g/L & 91 & $2.7 I(0.77)$ & $2.07 \pm 0.32$ & $3.06 \pm 0.55$ & $3.35 \pm 0.96$ & $<0.001$ \\
\hline D-dimer, ug/L & 87 & $204.69(298.59)$ & $218.11 \pm 409.26$ & $|74.59 \pm| 42.52$ & $258.85 \pm 305.62$ & 0.645 \\
\hline
\end{tabular}

Abbreviations: ECOG PS, Eastern Cooperative Oncology Group performance status; WBC, white blood cell count; HGB, hemoglobin; PLT, platelet count; NLR, neutrophil-to-lymphocyte ratio; PLR, platelet-to-lymphocyte ratio; TP, total protein; ALB, albumin; ALT, alanine aminotransferase; AST, aspartate aminotransferase; TBIL, total bilirubin; DBIL, direct bilirubin; TMZ, Temozolomide. 


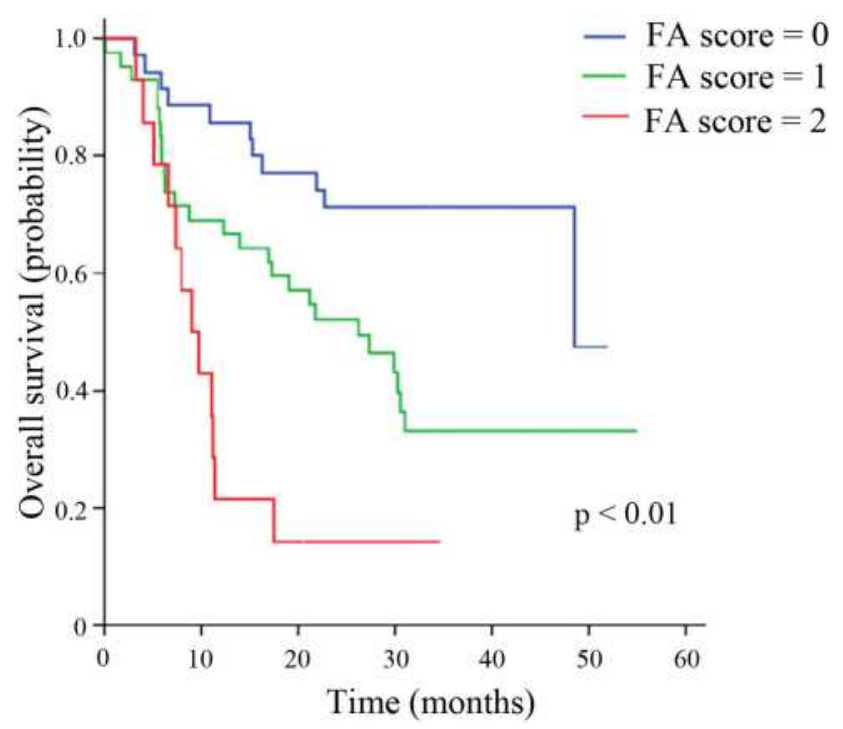

Figure I Kaplan-Meier survival curves comparing the three FA score groups.

\section{Results}

\section{Patient Characteristics}

Between November 2011 and August 2013, 91 patients were included in the final study population (Table 1). Regarding the FA score in patients, 35 (38.5\%) were classified with a FA score of $0,42(46.2 \%)$ were classified with a FA score of 1 , and $14(15.3 \%)$ were classified with a FA score of 2 . The median age was 45.42 years, and FA scores of the older group had an older mean age $(\mathrm{p}=$ 0.002). There were 56 males and 35 females. A total of $60(65.9 \%)$ patients were grade II and $31(34.1 \%)$ patients were grade III. The tumor location of $53(58.2 \%)$ patients

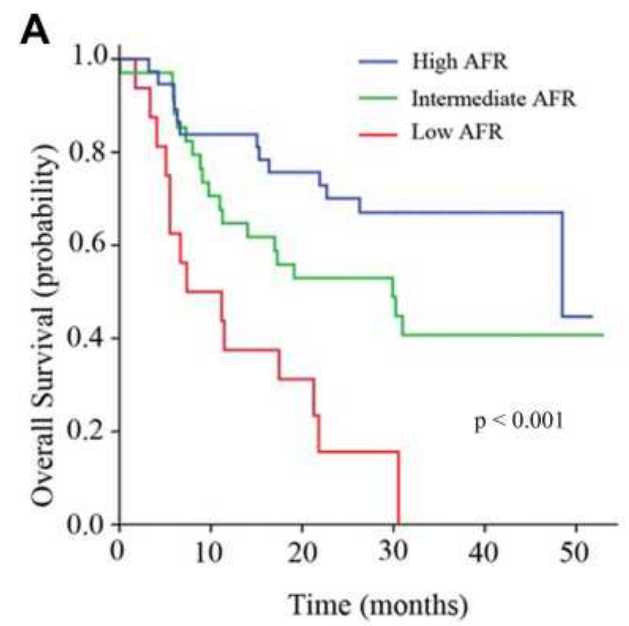

was the right brain, and the tumor location of the other patients was the left brain $(38,41.8 \%)$. There was no significant difference among the three FA groups in gender $(\mathrm{p}=0.648)$, ECOG PS $(\mathrm{p}=0.467)$, WHO grade $(\mathrm{p}=$ $0.082)$, tumor location $(\mathrm{p}=0.574)$, tumor size $(\mathrm{p}=$ $0.735)$, and adjuvant treatment after surgery $(\mathrm{p}=0.920)$.

The characteristics of total protein $(p=0.002)$, albumin $(p<$ $0.001)$, and fibrinogen $(p<0.001)$ were significantly different among three FA groups. The FA score 1 group had the highest total protein $(68.47 \pm 5.94 \mathrm{~g} / \mathrm{L})$, the FA score 0 group had the highest albumin $(42.71 \pm 2.69 \mathrm{~g} / \mathrm{L})$, and the FA score 2 group had the highest fibrinogen level $(3.35 \pm 0.96 \mathrm{~g} / \mathrm{L})$ (Table 1).

\section{Survival Analysis}

On the last follow-up date, 49 (53.8\%) patients had died. The median OS of all patients was 29.90 months [95\% confidence interval (CI): 21.31-38.49]. The FA score was significantly associated with the OS $(\mathrm{p}<0.001)$ (Figure 1$)$. The patients with the highest FA score (FA score =2) had the worst OS (9.1 months, 95\% CI: 5.8-12.3). The median OS for FA scores 0 and 1 were 39.6 months $(95 \% \mathrm{CI}$ : 33.6-45.7) and 26.3 months (95\% CI: $14.2-38.3)$, respectively (Subgroup analysis for grades II and III please see Supplementary Figures 1 and 2).

As shown in Figure 2A, the AFR was positively associated with the OS $(p<0.001)$. Further comparative analyses showed that the area under curves (AUCs) of FA scores $(0.71,95 \%$ CI: $0.60-0.82)$ was higher than the AFR (0.69; 95\% CI: 0.58-0.80) (Figure 2B). We therefore selected the FA score in subsequent survival analyses.

B

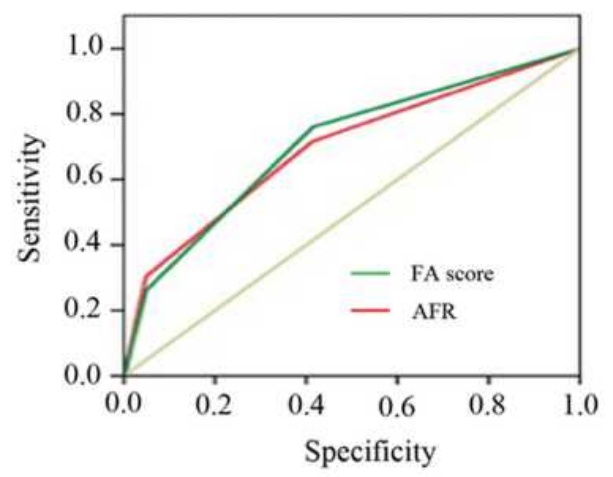

Figure 2 The comparative analyses between FA score and AFR. (A). Kaplan-Meier survival curves comparing the three albumin/fibrinogen ratio (AFR) score groups. (B). Table I. Relationships between patient demographics and clinicopathological characteristics and FA score with characteristics. 
Table 2 The Result of Univariate Cox Hazards Analysis of Overall Survival

\begin{tabular}{|c|c|c|c|}
\hline Variables & HR & $95 \% \mathrm{Cl}$ & $P$ value \\
\hline $\begin{array}{l}\text { Age } \\
\qquad \begin{array}{l}<44 \\
\geq 44\end{array}\end{array}$ & 2.837 & $1.555-5.178$ & 0.001 \\
\hline $\begin{array}{l}\text { Gender } \\
\text { Male } \\
\text { Female }\end{array}$ & 0.810 & $0.446-1.473$ & 0.491 \\
\hline $\begin{array}{c}\text { ECOG PS } \\
0-1 \\
2-3\end{array}$ & 2.271 & $1.28 \mid-4.024$ & 0.005 \\
\hline $\begin{array}{c}\text { Grade } \\
\text { II } \\
\text { III }\end{array}$ & 2.948 & $1.668-5.213$ & $<0.001$ \\
\hline $\begin{array}{l}\text { Location } \\
\text { Left brain } \\
\text { Right brain }\end{array}$ & 0.711 & $0.403-1.256$ & 0.240 \\
\hline $\begin{array}{l}\text { Max tumor } \\
\quad<4.8 \mathrm{~cm} \\
\geq 4.8 \mathrm{~cm}\end{array}$ & 0.924 & $0.520-1.639$ & 0.786 \\
\hline Adjuvant treatment & 1.103 & $0.921-1.321$ & 0.288 \\
\hline $\begin{array}{l}\text { WBC } \\
\qquad 8.7 * 10^{9} / \mathrm{L} \\
\geq 8.7 * 10^{9} / \mathrm{L}\end{array}$ & 2.390 & I.275-4.482 & 0.007 \\
\hline $\begin{array}{l}\text { HGB } \\
\qquad \begin{array}{l}<132 \mathrm{~g} / \mathrm{L} \\
\geq 132 \mathrm{~g} / \mathrm{L}\end{array}\end{array}$ & 1.764 & $0.878-3.545$ & 0.111 \\
\hline $\begin{array}{l}\text { PLT } \\
\qquad 203^{*} 10^{9} / \mathrm{L} \\
\quad \geq 203^{*} 10^{9} / \mathrm{L}\end{array}$ & 0.665 & $0.376-1.174$ & 0.159 \\
\hline $\begin{array}{l}\text { NLR } \\
\quad<4.1 \\
\geq 4.1\end{array}$ & 2.318 & $1.219-4.410$ & 0.010 \\
\hline $\begin{array}{l}\text { PLR } \\
\qquad \begin{array}{l}<117.4 \\
\geq 117.4\end{array}\end{array}$ & 1.345 & $0.767-2.359$ & 0.302 \\
\hline $\begin{array}{l}\text { TP } \\
\qquad 61.6 \mathrm{~g} / \mathrm{L} \\
\geq 61.6 \mathrm{~g} / \mathrm{L}\end{array}$ & 0.613 & $0.274-1.373$ & 0.234 \\
\hline $\begin{array}{l}\text { ALB } \\
\qquad \begin{array}{l}<38.3 \mathrm{~g} / \mathrm{L} \\
\geq 38.3 \mathrm{~g} / \mathrm{L}\end{array}\end{array}$ & 0.449 & $0.242-0.834$ & 0.011 \\
\hline $\begin{array}{l}\text { ALT } \\
\quad<20 \mathrm{U} / \mathrm{L} \\
\quad \geq 20 \mathrm{U} / \mathrm{L}\end{array}$ & 1.809 & $1.004-3.261$ & 0.049 \\
\hline
\end{tabular}

(Continued)
Table 2 (Continued).

\begin{tabular}{|l|c|c|c|}
\hline Variables & HR & 95\% CI & P value \\
\hline $\begin{array}{c}\text { AST } \\
<16 \mathrm{U} / \mathrm{L} \\
\geq 16 \mathrm{U} / \mathrm{L}\end{array}$ & 0.782 & $0.430-1.423$ & 0.421 \\
\hline $\begin{array}{c}\text { TBIL } \\
<6.8 \mathrm{umol} / \mathrm{L} \\
\geq 6.8 \mathrm{umol} / \mathrm{L}\end{array}$ & 0.686 & $0.329-1.427$ & 0.313 \\
\hline $\begin{array}{c}\text { DBIL } \\
<2.9 \mathrm{umol} / \mathrm{L} \\
\geq 2.9 \mathrm{umol} / \mathrm{L}\end{array}$ & 0.648 & $0.360-1.165$ & 0.147 \\
\hline $\begin{array}{c}\text { Fibrinogen } \\
<2.5 \mathrm{~g} / \mathrm{l} \\
\geq 2.5 \mathrm{~g} / \mathrm{l}\end{array}$ & 3.155 & $1.659-6.000$ & $<0.001$ \\
\hline $\begin{array}{c}\text { D-dimer } \\
<106 \mathrm{ug} / \mathrm{L} \\
\geq 106 \mathrm{ug} / \mathrm{L}\end{array}$ & & & \\
\hline $\begin{array}{c}\text { FA } \\
0 \\
\text { I } \\
2\end{array}$ & $2.44 \mathrm{I}$ & $1.604-3.715$ & $<0.001$ \\
\hline
\end{tabular}

Abbreviations: ECOG PS, Eastern Cooperative Oncology Group performance status; WBC, white blood cell count; HGB, hemoglobin; PLT, platelet count; NLR, neutrophil-to-lymphocyte ratio; PLR, platelet-to-lymphocyte ratio; TP, total protein; ALB, albumin; ALT, alanine aminotransferase; AST, aspartate aminotransferase; TBIL, total bilirubin; DBIL, direct bilirubin; FA, fibrinogen and albumin.

Table 3 The Result of Multivariate Cox Hazards Analysis of Overall Survival

\begin{tabular}{|l|c|c|c|}
\hline Variable & HR & $95 \%$ Cl & P value \\
\hline Age & 3.014 & $1.522-5.969$ & 0.002 \\
WBC & 4.243 & $2.078-8.666$ & $<0.001$ \\
FA & 1.916 & $1.205-3.048$ & 0.006 \\
\hline
\end{tabular}

Abbreviations: WBC, white blood cell count; FA, fibrinogen and albumin.

\section{A Predictive Nomogram for the Prognoses of Patients with Grade II/III Glioma}

Univariate and multivariate analyses were used to show that three covariates (FA score, age, and white blood count) had significant correlations with the OS (Tables 2 and 3). Figure 3 shows the predictive nomogram of OS from the multivariate analyses. The c-index was 0.783 (95\% CI: 0.72-0.84), indicating good performance of predicting OS for patients with Grade II/III gliomas. The calibration curves for 1- and 3-year survival patients also 


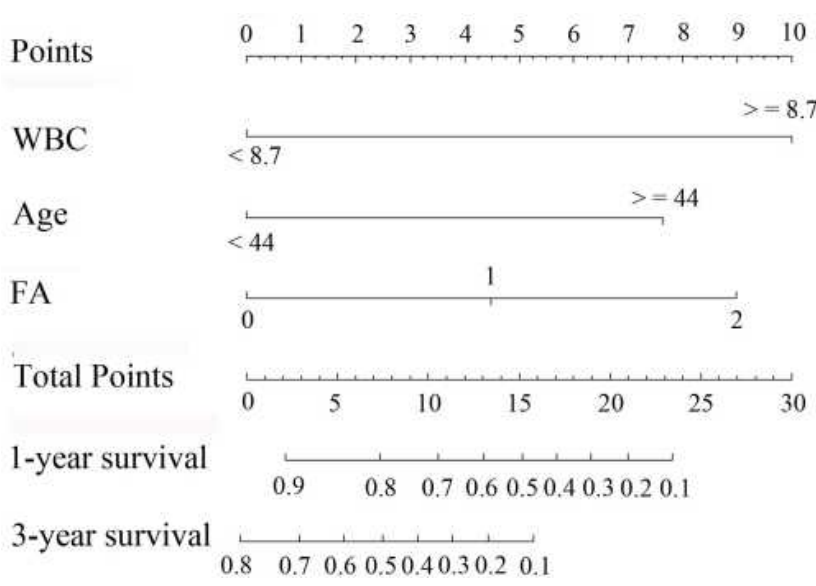

Figure $3 \mathrm{~A}$ nomogram predicting I-year and 3-year overall survival in patients with WHO Grade II/III gliomas.

showed the accurate predictive ability of the nomogram (Figure 4A and B). The time-dependent ROC curve was calculated for the nomogram for every month (Figure 4C).

\section{Discussion}

In recent years, an increasing number of studies have focused on the prognosis of GBM, because of its high incidence and prevalence. ${ }^{17-19}$ However, LGGs (WHO Grade II/III gliomas) represent important causes of morbidity and mortality in the young adult population..$^{20}$ Some past and ongoing clinical trials (NCT02766270, NCT01164189, and NCT03906448) have also focused on LGG patients. ${ }^{21}$ Furthermore, it is necessary to identify prognostic factors to optimize treatment for patients with LGGs. In the present study, we showed that the FA score was significantly associated with OS in patients with LGGs, and we developed a nomogram to provide the survival probability of individual patients.

The plasma fibrinogen and albumin level are the most commonly used indicators of coagulation and nutritional status, respectively. Ways to combine fibrinogen and albumin levels, including the AFR and FA score, were reported as prognostic markers in some solid tumors, including HGGs. ${ }^{11,13,22-26}$ In the present study, the ROC curve showed that FA scores had a higher sensitivity and specificity than AFR for predicting OS. We showed for the first time a higher FA score was significantly associated with a worse OS, which was an independent prognostic factor in LGGs patients. The exact mechanism for these associations remains unclear. It is well-known that fibrinogen is an acute-phase reactant of systemic inflammation, which includes CRP, leucocytes, ferritin, thrombocytes, and fibrinogen. ${ }^{27,28}$ Fibrinogen plays a major role in tumorrelated biological behaviors and provides a stable framework for the extracellular matrix of tumors, thus, promoting cancer cell adhesion, migration, and invasion. ${ }^{29}$ Albumin is a biomarker of the systemic inflammation response (SIR) and reflects malnutrition and immune ability. ${ }^{30-32}$ Some studies have shown that low levels of albumin might be a potential poor prognostic biomarker for gliomas. ${ }^{33,34}$ Possible reasons considered to explain this phenomenon are as follows. First, fibrinogen may regulate the SIR by producing tumor-released inflammatory factors such as cytokines interleukin-1, interleukin-6, tumor necrosis factor- $\alpha$ (TNF- $\alpha$ ), vascular endothelial growth factor (VEGF), fibroblast growth factor-2, and platelet-derived growth factor. ${ }^{35-37}$ IL-6 and TNF- $\alpha$ can suppress synthesis of albumin and VEGF can induce vascular permeability, which lead to a decrease in serum albumin levels. ${ }^{36,38}$ Second, malnutrition due to serum albumin levels increases the chance of infection and promotes the development of malignant tumors by accelerating SIR. ${ }^{39}$

Numerous recent studies have provided evidence that some other SIR biomarkers, including the neutrophil-to-
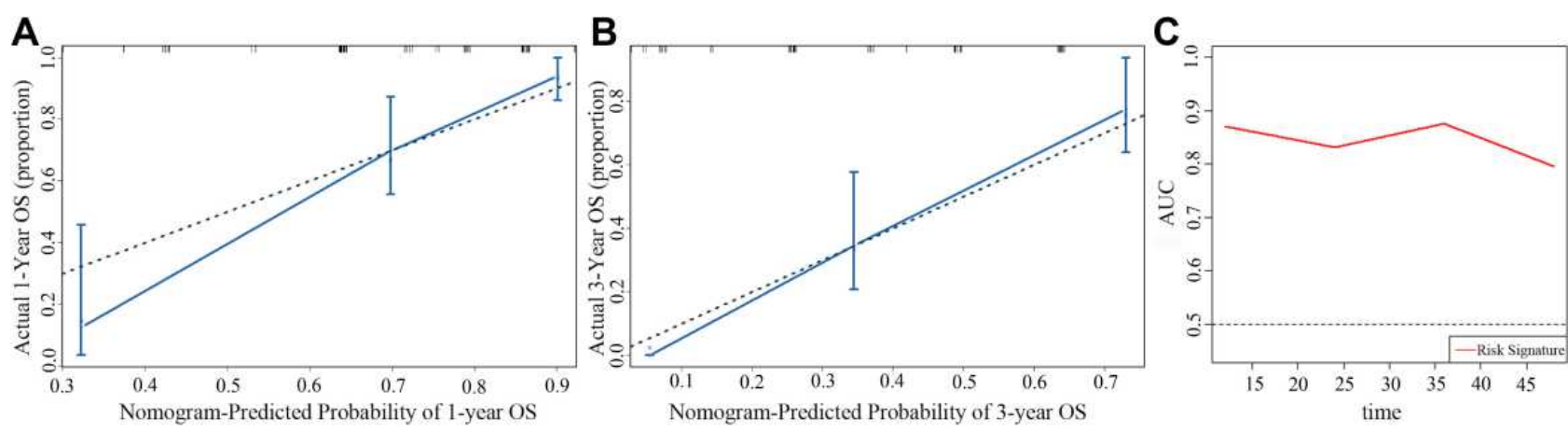

Figure 4 Validation of the nomogram. (A). The calibration curve of predicting patient survival percentages at I year. (B). The calibration curve of predicting patient survival percentages at 3 year. (C). The integrated area under the curve was calculated for the nomogram for every month. 
lymphocyte ratio (NLR) and platelet-to-lymphocyte ratio (PLR), are related to the survival of gastric cancer patients. ${ }^{40-44}$ In the present study, we also evaluated the prognostic values of PLR and NLR. The results showed that PLR was not an independent predictive index of LGGs, which was consistent with some previous reports in gliomas. ${ }^{45,46} \mathrm{~A}$ recent meta-analysis of 18 studies containing 3261 patients showed that NLR [hazard ratio (HR): 1.38; 95\% CI: $1.09-1.74 ; \mathrm{p}=0.008]$ was one of the independent indices for predicting the OS of gliomas. ${ }^{45}$ In the present study, elevated NLR was statistically significant in univariate analysis but not multivariate analysis. The possible reason was the white blood count was also an independent prognostic factor in LGGs patients in this study. Its large prognostic effect weakened the predictive effect of other leukocyte components.

The nomogram provided a graphic representation of various risk factors and estimated survival probabilities tailored to individual patients. ${ }^{47,48}$ The score was obtained based on the result of each variable for each patient by locating the corresponding scale of the variable. The total values were then added, and a vertical line was drawn through the survival scales that provided the probability for a 1 - and 3-year OS. This nomogram can effectively promote communication between doctors and patients, to help them select more beneficial treatment options. Our nomogram relied on easily available parameters from clinical-related and hematological characteristics and achieved an AUC value $>0.8$ for survival prediction. In addition, a good c-index and calibration curve will support the accuracy of the prediction nomogram.

The present study had some limitations. First, this was a retrospective, single center study. A multi-center study with a larger sample size would have been more representative. Second, the nomogram lacked validation cohorts. More patients are needed to establish the internal and external validation cohorts. Third, because the status of tumor recurrence was not accurate and complete, disease free survival data were not analyzed in this study. Fourth, the lack of information on molecular markers (such as IDH status, $1 \mathrm{p} / 19 \mathrm{q}$ LOH status, and MGMT promoter methylation status) and postoperative treatment regimens, limited further in-depth analyses.

\section{Conclusions}

The FA score was an independent risk factor for OS in LGG patients. A reliable nomogram has great potential application in clinical practice for estimating the mortality risk in treating LGG patients on an individual basis.

\section{Acknowledgments}

This work was supported by the National Natural Science Foundation of China (No.81802500), Natural Science Foundation of Liaoning Province (No.20180550817) and 345 Talent Project of Shengjing Hospital.

\section{Disclosure}

The authors report no conflicts of interest in this work.

\section{References}

1. Ostrom QT, Gittleman H, Fulop J, et al. CBTRUS statistical report: primary brain and central nervous system tumors diagnosed in the United States in 2008-2012. Neuro Oncol. 2015;17(Suppl 4):iv1iv62. doi:10.1093/neuonc/nov189

2. Walsh KM, Ohgaki H, Wrensch MR. Epidemiology. Handb Clin Neurol. 2016;134:3-18.

3. Ostrom QT, Gittleman H, Liao P, et al. CBTRUS statistical report: primary brain and central nervous system tumors diagnosed in the United States in 2007-2011. Neuro Oncol. 2014;16(Suppl 4):iv1-63. doi:10.1093/neuonc/nou223

4. Abrigo JM, Fountain DM, Provenzale JM, et al. Magnetic resonance perfusion for differentiating low-grade from high-grade gliomas at first presentation. Cochrane Database Syst Rev. 2018;1:CD011551. doi:10.1002/14651858.CD011551.pub2

5. Kleihues P, Louis DN, Scheithauer BW, et al. The WHO classification of tumors of the nervous system. J Neuropathol Exp Neurol. 2002;61(3):215-219. doi:10.1093/jnen/61.3.215

6. Wirsching HG, Galanis E, Weller M. Glioblastoma. Handb Clin Neurol. 2016;134:381-397.

7. Ding H, Huang Y, Li Z, et al. Prediction of IDH status through MRI features and enlightened reflection on the delineation of target volume in low-grade gliomas. Technol Cancer Res Treat. 2019;18:1533033819877167. doi:10.1177/1533033819877167

8. Pouratian N, Schiff D. Management of low-grade glioma. Curr Neurol Neurosci Rep. 2010;10(3):224-231. doi:10.1007/s11910010-0105-7

9. Stupp R, Brada M, van den Bent MJ, Tonn JC, Pentheroudakis G; Group EGW. High-grade glioma: ESMO clinical practice guidelines for diagnosis, treatment and follow-up. Ann Oncol. 2014;25(Suppl 3): iii93-101. doi:10.1093/annonc/mdu050

10. Forrest LM, McMillan DC, McArdle CS, Angerson WJ, Dunlop DJ. Evaluation of cumulative prognostic scores based on the systemic inflammatory response in patients with inoperable non-small-cell lung cancer. $B r J$ Cancer. 2003;89(6):1028-1030. doi:10.1038/sj. bjc. 6601242

11. Matsuda S, Takeuchi H, Kawakubo H, et al. Cumulative prognostic scores based on plasma fibrinogen and serum albumin levels in esophageal cancer patients treated with transthoracic esophagectomy: comparison with the Glasgow prognostic score. Ann Surg Oncol. 2015;22(1):302-310. doi:10.1245/s10434-014-3857-5

12. He ZQ, Ke C, Al-Nahari F, et al. Low preoperative prognostic nutritional index predicts poor survival in patients with newly diagnosed high-grade gliomas. J Neurooncol. 2017;132(2):239-247. doi:10.1007/s11060-016-2361-0

13. Chan DY, Syn NL, Yap R, et al. Conversion surgery post-intraperitoneal paclitaxel and systemic chemotherapy for gastric cancer carcinomatosis peritonei. are we ready? J Gastrointest Surg. 2017;21(3):425-433. doi:10.1007/s11605-016-3336-3 
14. Mantovani A, Allavena P, Sica A, Balkwill F. Cancer-related inflammation. Nature. 2008;454(7203):436-444. doi:10.1038/ nature 07205

15. Qureshi YA, Sarker SJ, Walker RC, Hughes SF. Proximal resection margin in ivor-lewis oesophagectomy for cancer. Ann Surg Oncol. 2017;24(2):569-577. doi:10.1245/s10434-016-5510-y

16. Gan Y, Zhou X, Niu X, et al. Neutrophil/lymphocyte ratio is an independent prognostic factor in elderly patients with high-grade gliomas. World Neurosurg. 2019;127:e261-e267. doi:10.1016/j. wneu.2019.03.085

17. Armocida D, Pesce A, Di Giammarco F, Frati A, Santoro A, Salvati M. Long term survival in patients suffering from glio-blastoma multiforme: a single-center observational cohort study. Diagnostics (Basel). 2019;9(4). doi:10.3390/diagnostics9040209

18. Dietterle J, Wende T, Wilhelmy F, et al. The prognostic value of peri-operative neurological performance in glioblastoma patients. Acta Neurochir (Wien). 2019;162:417-425. doi:10.1007/s00701019-04136-4

19. Marques C, Carvelli J, Biard L, et al. Prognostic factors in anti-glomerular basement membrane disease: a multicenter study of 119 patients. Front Immunol. 2019;10:1665. doi:10.3389/fimmu.2019.01665

20. Picca A, Berzero G, Sanson M. Current therapeutic approaches to diffuse grade II and III gliomas. Ther Adv Neurol Disord. 2018;11:1756285617752039. doi:10.1177/1756285617752039

21. Seystahl K, Wiestler B, Hundsberger T, et al. Bevacizumab alone or in combination with irinotecan in recurrent WHO grade II and grade III gliomas. Eur Neurol. 2013;69(2):95-101. doi:10.1159/000343811

22. Chen S, Yan H, Du J, et al. Prognostic significance of pre-resection albumin/fibrinogen ratio in patients with non-small cell lung cancer: a propensity score matching analysis. Clin Chim Acta. 2018;482:203-208. doi:10.1016/j.cca.2018.04.012

23. Liang Y, Wang W, Que Y, et al. Prognostic value of the fibrinogen/ albumin ratio (FAR) in patients with operable soft tissue sarcoma. BMC Cancer. 2018;18(1):942. doi:10.1186/s12885-018-4856-X

24. Wang Y, Chen W, Hu C, et al. Albumin and fibrinogen combined prognostic grade predicts prognosis of patients with prostate cancer. $J$ Cancer. 2017;8(19):3992-4001. doi:10.7150/jca.21061

25. Li SQ, Jiang YH, Lin J, et al. Albumin-to-fibrinogen ratio as a promising biomarker to predict clinical outcome of non-small cell lung cancer individuals. Cancer Med. 2018;7(4):1221-1231. doi: $10.1002 / \mathrm{cam} 4.1428$

26. Ying J, Zhou D, Gu T, Huang J, Liu H. Pretreatment albumin/ fibrinogen ratio as a promising predictor for the survival of advanced non small-cell lung cancer patients undergoing first-line platinum-based chemotherapy. BMC Cancer. 2019;19(1):288. doi:10.1186/s12885-019-5490-y

27. Simpson-Haidaris PJ, Rybarczyk B. Tumors and fibrinogen. The role of fibrinogen as an extracellular matrix protein. Ann N Y Acad Sci. 2001;936:406-425. doi:10.1111/j.1749-6632.2001.tb03525.x

28. Gulen ST, Karadag F, Karul AB, et al. Adipokines and systemic inflammation in weight-losing lung cancer patients. Lung. 2012;190 (3):327-332. doi:10.1007/s00408-011-9364-6

29. Palumbo JS, Talmage KE, Massari JV, et al. Platelets and fibrin(ogen) increase metastatic potential by impeding natural killer cell-mediated elimination of tumor cells. Blood. 2005;105(1):178-185. doi:10.1182/blood-2004-06-2272

30. Esmon CT. Does inflammation contribute to thrombotic events? Haemostasis. 2000;30(Suppl 2):34-40. doi:10.1159/000054161

31. Dolan RD, Laird BJA, Klepstad P, et al. An exploratory study examining the relationship between performance status and systemic inflammation frameworks and cytokine profiles in patients with advanced cancer. Medicine (Baltimore). 2019;98(37):e17019. doi:10.1097/MD.0000000000017019
32. Xu Q, Yan Y, Gu S, et al. A novel inflammation-based prognostic score: the fibrinogen/albumin ratio predicts prognoses of patients after curative resection for hepatocellular carcinoma. J Immunol Res. 2018;2018:4925498. doi:10.1155/2018/4925498

33. Wang PF, Meng Z, Song HW, et al. Preoperative changes in hematological markers and predictors of glioma grade and survival. Front Pharmacol. 2018;9:886. doi:10.3389/fphar.2018.00886

34. Han S, Huang Y, Li Z, Hou H, Wu A. The prognostic role of preoperative serum albumin levels in glioblastoma patients. $B M C$ Cancer. 2015;15:108. doi:10.1186/s12885-015-1125-0

35. Reynes G, Vila V, Martin M, et al. Circulating markers of angiogenesis, inflammation, and coagulation in patients with glioblastoma. J Neurooncol. 2011;102(1):35-41. doi:10.1007/s11060-010-0290-х

36. Loeffler S, Fayard B, Weis J, Weissenberger J. Interleukin-6 induces transcriptional activation of vascular endothelial growth factor (VEGF) in astrocytes in vivo and regulates VEGF promoter activity in glioblastoma cells via direct interaction between STAT3 and Sp1. Int $J$ Cancer. 2005;115(2):202-213. doi:10.1002/ijc.20871

37. Albrecht U, Yang X, Asselta R, et al. Activation of NF-kappaB by IL-1beta blocks IL-6-induced sustained STAT3 activation and STAT3-dependent gene expression of the human gamma-fibrinogen gene. Cell Signal. 2007;19(9):1866-1878. doi:10.1016/j. cellsig.2007.04.007

38. Rothschild MA, Oratz M, Schreiber SS. Serum albumin. Hepatology. 1988;8(2):385-401. doi:10.1002/hep.1840080234

39. Li SQ, You XH, Sun F, et al. Albumin to fibrinogen ratio and fibrinogen to pre-albumin ratio are economical, simple and promising prognostic factors for solid malignancy. J Thorac Dis. 2019;11(Suppl 15):S2036-S2038. doi:10.21037/jtd.2019.08.96

40. Diakos CI, Charles KA, McMillan DC, Clarke SJ. Cancer-related inflammation and treatment effectiveness. Lancet Oncol. 2014;15 (11):e493-503. doi:10.1016/S1470-2045(14)70263-3

41. Wang J, Qu J, Li Z, et al. A prognostic model in metastatic or recurrent gastric cancer patients with good performance status who received first-line chemotherapy. Transl Oncol. 2016;9(3):256-261. doi:10.1016/j.tranon.2016.04.004

42. Lee S, Oh SY, Kim SH, et al. Prognostic significance of neutrophil lymphocyte ratio and platelet lymphocyte ratio in advanced gastric cancer patients treated with FOLFOX chemotherapy. BMC Cancer. 2013;13:350. doi:10.1186/1471-2407-13-350

43. Xin-Ji Z, Yong-Gang L, Xiao-Jun S, Xiao-Wu C, Dong Z, Da-jian Z. The prognostic role of neutrophils to lymphocytes ratio and platelet count in gastric cancer: a meta-analysis. Int J Surg. 2015;21:84-91. doi:10.1016/j.ijsu.2015.07.681

44. Wang J, Qu J, Li Z, et al. Pretreatment platelet-to-lymphocyte ratio is associated with the response to first-line chemotherapy and survival in patients with metastatic gastric cancer. J Clin Lab Anal. 2018;32 (1):e22185. doi:10.1002/jcla.22185

45. Wang DP, Kang K, Lin Q, Hai J. Prognostic significance of preoperative systemic cellular inflammatory markers in gliomas: a systematic review and meta-analysis. Clin Transl Sci. 2019;13:179-188. doi:10.1111/cts.12700

46. Yang T, Mao P, Chen X, et al. Inflammatory biomarkers in prognostic analysis for patients with glioma and the establishment of a nomogram. Oncol Lett. 2019;17(2):2516-2522. doi:10.3892/ ol.2018.9870

47. Madsen LT. Cancer prediction nomograms for advanced practitioners in oncology. J Adv Pract Oncol. 2014;5(5):380-382.

48. Giordano A, Egleston BL, Hajage D, et al. Establishment and validation of circulating tumor cell-based prognostic nomograms in first-line metastatic breast cancer patients. Clin Cancer Res. 2013;19(6):1596-1602. doi:10.1158/1078-0432.CCR-12-3137 


\section{Publish your work in this journal}

The International Journal of General Medicine is an international, peer-reviewed open-access journal that focuses on general and internal medicine, pathogenesis, epidemiology, diagnosis, monitoring and treatment protocols. The journal is characterized by the rapid reporting of reviews, original research and clinical studies across all disease areas. The manuscript management system is completely online and includes a very quick and fair peer-review system, which is all easy to use. Visit http://www.dovepress.com/ testimonials.php to read real quotes from published authors.

Submit your manuscript here: https://www.dovepress.com/international-journal-of-general-medicine-journal 